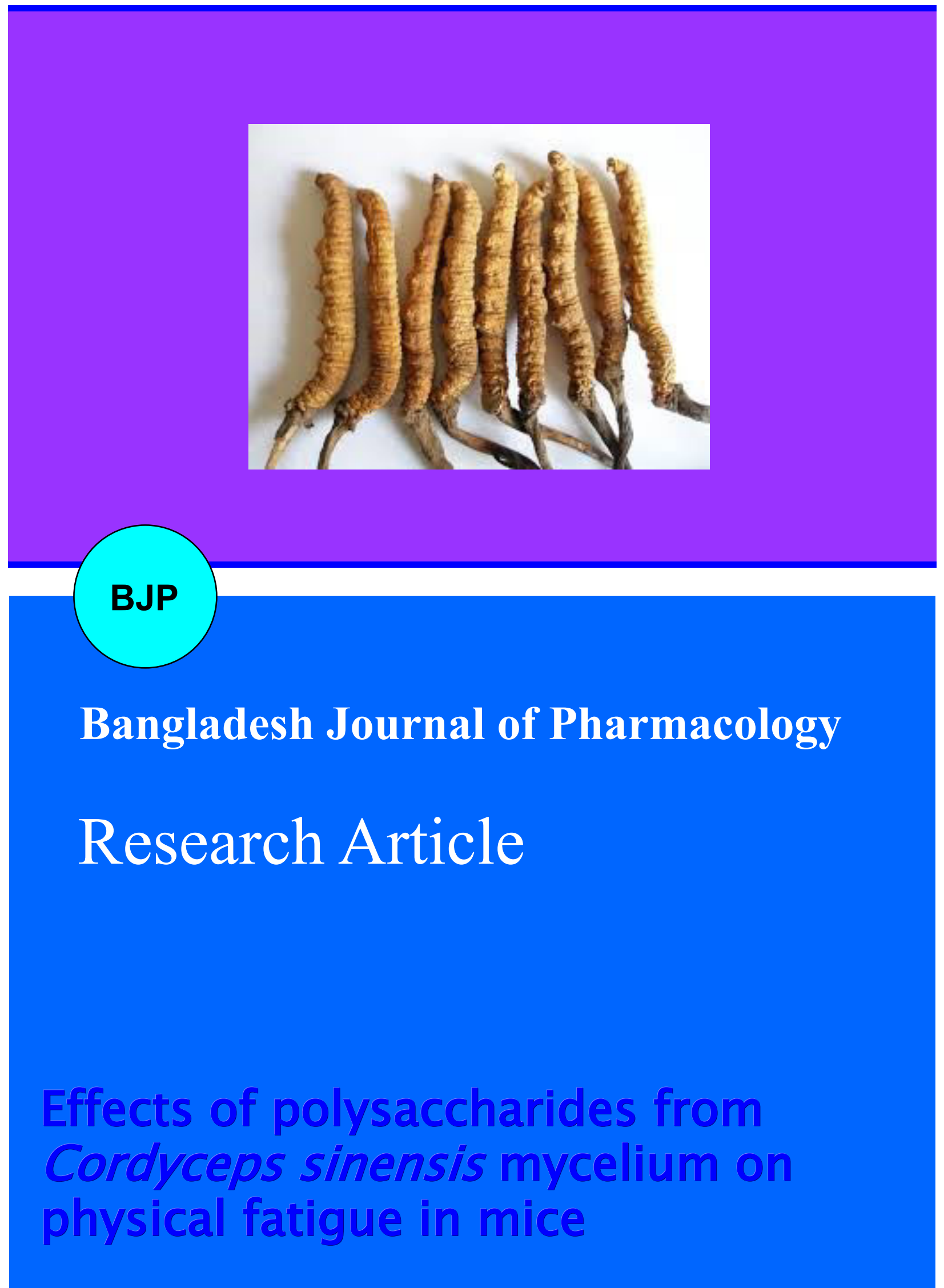




\title{
Effects of polysaccharides from Cordyceps sinensis mycelium on physical fatigue in mice
}

\author{
Feng Yan', Yan Zhang ${ }^{2}$ and Beibei Wang1 \\ ${ }^{1}$ Department of Physical Education, University of International Business and Economics, Beijing 10029, China; \\ ${ }^{2}$ Harbin Institute of Physical Education, Harbin 150008, China.
}

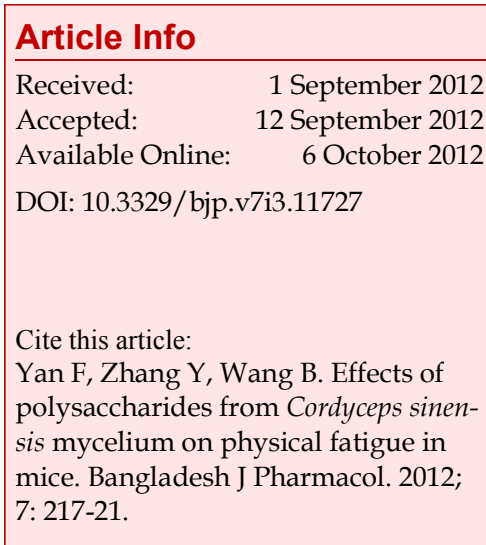

\begin{abstract}
This study was designed to determine the effects of polysaccharides from Cordyceps sinensis mycelium (CSP) on physical fatigue in mice. The mice were randomly divided into four groups ( $\mathrm{n}=16$ in each group), i.e. control group, low-dose CSP treated group, intermediate-dose CSP treated group and highdose CSP treated group. The mice in the treated groups received CSP (100, 200 and $400 \mathrm{mg} / \mathrm{kg}$, ig), and the mice in the control group received drinking water ig. After 28 days, a forced swimming test was performed and some biochemical parameters related to fatigue were examined. The present data suggested that CSP could extend the exhaustive swimming time of mice, as well as increase the hepatic glycogen and muscle glycogen levels, and decrease the blood lactic acid and BUN levels. These results indicated that CSP had anti-fatigue effects.
\end{abstract}

\section{Introduction}

Fatigue, the seventh most common symptom in primary health care, is one of the least understood conditions in medical science (Drel and Sybirna, 2006). Fatigue is best defined as the difficulty in initiating or sustaining voluntary activities (Ataka et al., 2009), and it can be classified as physical or mental, depending on its cause. Physical fatigue is caused by such things as forced exercise or swimming, and mental fatigue is caused by sleep, deprivation, etc. (Huang et al., 2011). Physical fatigue is thought to be accompanied by deterioration in performance (Evans and Lambert, 2007; Jin et al., 2009). Recent researches show that physical fatigue after exercise can be caused by various biochemical mechanisms, such as the depletion of energy stores, dysfunctions in cellular components that are responsible for producing energy, and the production of free radicals inside muscle cells (Ikeuchi et al., 2006; Yu et al., 2006; Xu and Wang, 2012). In the last few decades, health scholars and athletic physiologist have been looking for natural active products that not only can improve athletic ability, postpone fatigue and accelerate the elimination of fatigue in human beings, but also have few side effects.

Cordyceps sinensis, so called "winter-worm and summergrass," is an entomogenous fungus belonging to Ascomycota, Sordariomycetes, Hypocreales, Clavicipitaceae (Lindau) O.E. Erikss., Cordyceps (Fr.) Link ( Dong et al., 2009). C. sinensis has long been used in Chinese medicine to replenish the kidney and soothe the lung, for the treatment of fatigue, night sweating, hyposexualities, hyperglycemia, hyperlipidemia, asthenia after severe illness, respiratory disease, renal dysfunction and renal failure, arrhythmias and other heart diseases, and liver disease ( Chen et al., 2004; Li et al., 2005). Nowadays the medicinal value of $C$. sinensis has gained worldwide attention and attracted great research effort towards the scientific rediscovery of this herbal medicine. Recent scientific studies shows that $C$. sinensis contains crude protein, D-mannitol, cordycepin and polysaccharides (Ji et al., 2009). The polysaccharides from Cordyceps sinensis have been reported to exhibit a 
variety of biological activities, including anti-tumor, antiinflammatory, anti-oxidant, hypoglycemic and immunomodulatory properties (Li et al., 2005; Kuo et al., 2007; Wang et al., 2009; Zhang et al., 2011). However, there are few reports on its anti-fatigue properties. Therefore, the present study was to investigate the effects of polysaccharides from $C$. sinensis mycelium (CSP) on physical fatigue using a forced swimming test in mice and aimed to provide experimental basis for further study of CSP.

\section{Materials and Methods}

\section{Chemicals and reagents}

The detection kits for blood lactic acid, blood urea nitrogen (BUN), hepatic glycogen and muscle glycogen were purchased from Nanjing Jiancheng Bioengineering Institute (Nanjing, China). All other chemicals and reagents used were of analytical grade and MilliQ grade water was used.

\section{Preparation of polysaccharides from C. sinensis myce- lium}

The dried $C$. sinensis mycelium was purchased from Tong-Ren-Tang Pharmaceutical Group (Beijing, China) and authenticated by Yiyang Zhang, a biologist of Jilin University (Changchun, China). Preparation of polysaccharides from C. sinensis mycelium (CSP) was carried out according to previous report ( $\mathrm{Wu}$ et al., 2006). Dried C. sinensis mycelium were extracted with ethanol (95 and 85\%, respectively) to defat and decolorize, and then extracted with aqueous $75 \%$ ethanol overnight. After centrifugation (6700 rpm, $30 \mathrm{~min}$ ), the residue was dried naturally and then extracted with $0.05 \mathrm{M}$ phosphate buffer $(\mathrm{pH}=7.0)$ for $10 \mathrm{~h}$ at $80^{\circ} \mathrm{C}$, the process of extraction was repeated. After centrifugation (6700 rpm, $30 \mathrm{~min}$ ), the extracting liquids were combined, removed solvents under reduced pressure and dialyzed. The nondialyzable phase was diluted with 95\%ethanol, and the resulting precipitate was collected by centrifugation, washed three times with acetone, and dried. The yield of CSP was 3.7\%.

\section{Animals and grouping}

Male Kunming mice (18 to $22 \mathrm{~g}$ ) were purchased from Vital River Lab Animal Technology Co., Ltd. (Beijing, China) and they were maintained in polypropylene cages at a temperature of $22 \pm 1^{\circ} \mathrm{C}$ and relative humidity of $50 \pm 5 \%$ in clean environment under 12:12 hours light-dark cycle. Standard pellets (purchased from Xincaihong Feed Co., Ltd., Beijing, China) and water was provided ad libitum. The experiments were carried out according to the "Principles of Laboratory Animal Care" (World Health Organization Chronicle, 1985). After one week of acclimatization, the animals were randomly divided into four groups $(n=16$ in each group), i.e. control (C) group, low-dose CSP treated (LC) group, intermediate-dose CSP treated (IC) group and high-dose CSP treated (HC) group. The mice in the treated groups received CSP (100, 200 and $400 \mathrm{mg} / \mathrm{kg}$, ig), and the mice in the control group received drinking water ig for 28 days.

\section{Forced swimming test}

The physical fatigue was induced by forcing animals to swim until exhaustion. After 28 days, eight mice were taken out from each group for the forced swimming test. The procedure used was described previously (Jung et al., 2004; Kamakura et al., 2005) with some modifications. Briefly, $30 \mathrm{~min}$ after the last treatment, the mice were placed individually in an acrylic plastic tank $(50 \times 50 \times 40 \mathrm{~cm})$ with $30 \mathrm{~cm}$ depth of water maintained at $25 \pm 1^{\circ} \mathrm{C}$. A tin wire ( $7 \%$ of body weight) was loaded on the tail root of each mouse. The mice were assessed to be exhausted when they failed to rise to the surface of water to breathe within a $10 \mathrm{~s}$ period, and the swimming time was immediately recorded.

\section{Determination of some biochemical parameters related to fatigue}

After 28 days, the other eight mice were taken out from each group for analyses of some biochemical parameters related to fatigue. $30 \mathrm{~min}$ after the last treatment, the mice were forced to swim for 90 min without a load, and then the blood were collected through eyeball to prepare the serum for use. Meanwhile, the liver and gastrocnemius muscle were collected to be made into $10 \%$ homogenates with normal saline at $4^{\circ} \mathrm{C}$ as soon as possible. The levels of blood lactic acid, blood urea nitrogen (BUN), hepatic glycogen and muscle glycogen were tested following the recommended procedures provided by the kits.

\section{Data analysis}

The data were expressed as mean \pm SD. Statistical analysis was performed by one-way ANOVA followed by least-significant difference (LSD). A difference was considered significant when $\mathrm{p}<0.05$.

\section{Results and Discussion}

The forced swimming test has been used to evaluate the anti-fatigue effects of medicine, since this apparatus work well for evaluating the endurance capacity of mice and gives a high reproducibility. The length of the exhaustive swimming time indicate the degree of fatigue (Zhang et al., 2010; Yu et al., 2010). As shown in Figure 1, The exhaustive swimming time of mice in the LC, IC and HC groups was significantly prolonged compared with that in the $C$ group $(p<0.05)$, which was 1.3, 1.8 and 1.9 times longer than that in the $C$ group, respectively. The results indicated that CSP had anti- 


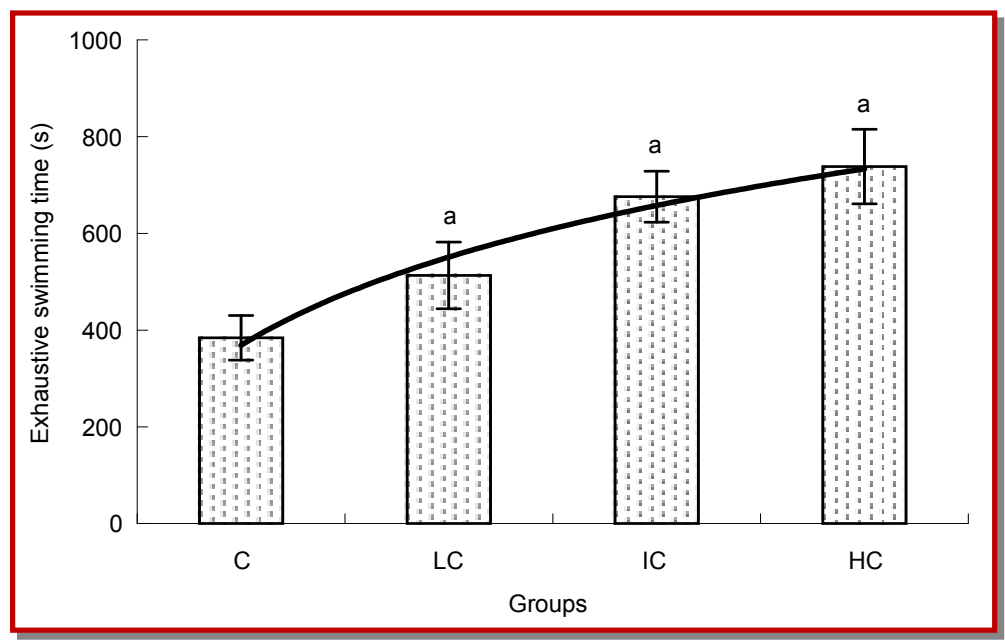

Figure 1: The effect of CSP on the exhaustive swimming time of mice. Data are expressed as mean \pm S.D. a $<<0.05$ compared with control group

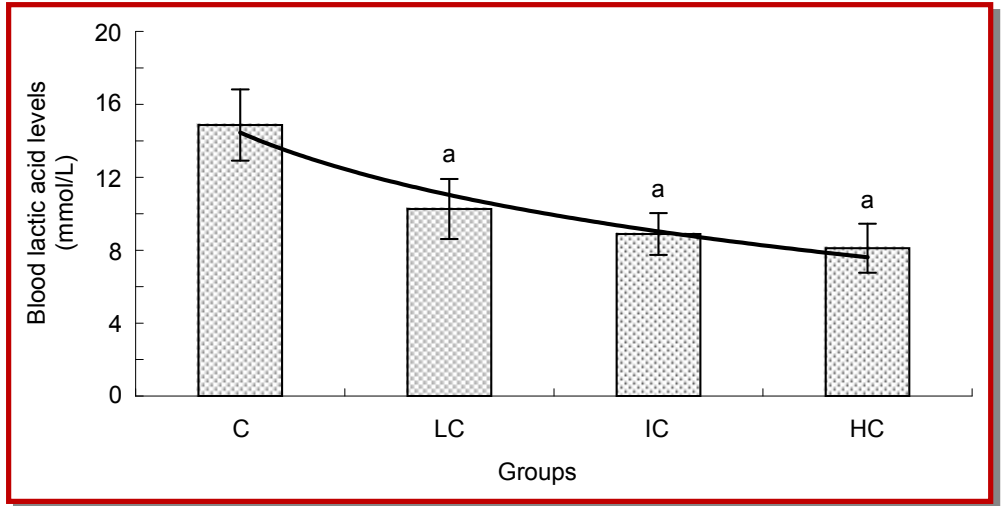

Figure 2: The effects of CSP on the blood lactic acid levels of mice. Data are expressed as mean \pm S.D. ap $<0.05$ compared with control group

fatigue effects and these effects were dose-dependent.

Blood lactic acid, blood urea nitrogen, hepatic glycogen and muscle glycogen are some biochemical parameters related to fatigue. Blood lactic acid is the glycolysis product of carbohydrate under an anaerobic condition, and glycolysis is the main energy source for fierce exercise in a short time. Therefore, blood lactic acid is closely related to workload intensity and is one of the important indicators for judging the intensity of the exercise or the degree of fatigue. In other words, blood lactic acid represents the degree of fatigue after exercise and the condition of recovery (Wang et al., 2006). As shown in Figure 2, The blood lactic acid levels of mice in the LC, IC and HC groups were significantly lower compared with that in the $C$ group $(p<0.05)$. The results indicated that CSP could effectively retard and lower the blood lactic produced, postpone the appearance of fatigue.

Blood urea nitrogen (BUN), the metabolic outcome of protein and amino acid, is a sensitive index to evaluate the bearing capability when body suffer from a physical load (Tan et al., 2012). Protein and amino acids have a stronger katabolic metabolism when body cannot obtain enough energy by sugar and fat catabolic metabolism. There is a positive correlation between the urea nitrogen in vivo and the exercise tolerance (Zhang et al., 2010). Therefore, BUN is another sensitive indicator of fatigue status. As shown in Figure 3, The BUN levels of mice in the IC and HC groups were significantly lower compared with that in the $\mathrm{C}$ group $(p<0.05)$. Although the BUN levels of mice in the LC group were also decreased, no significant difference was observed $(p>0.05)$. The results indicated that CSP might reduce catabolic decomposition of protein for energy, which was indicative of enhanced endurance.

Glycogen is the important resource of energy during exercise, and the role of glycogen is to complement the consumption of blood glucose and maintain the blood glucose in the physiologic range (Ding et al., 2011). Fatigue will happen when the glycogen is mostly consumed. So glycogen is a sensitive indicator to test fatigue and increasing the glycogen storage conduces to enhancing the endurance capacity and locomotory 


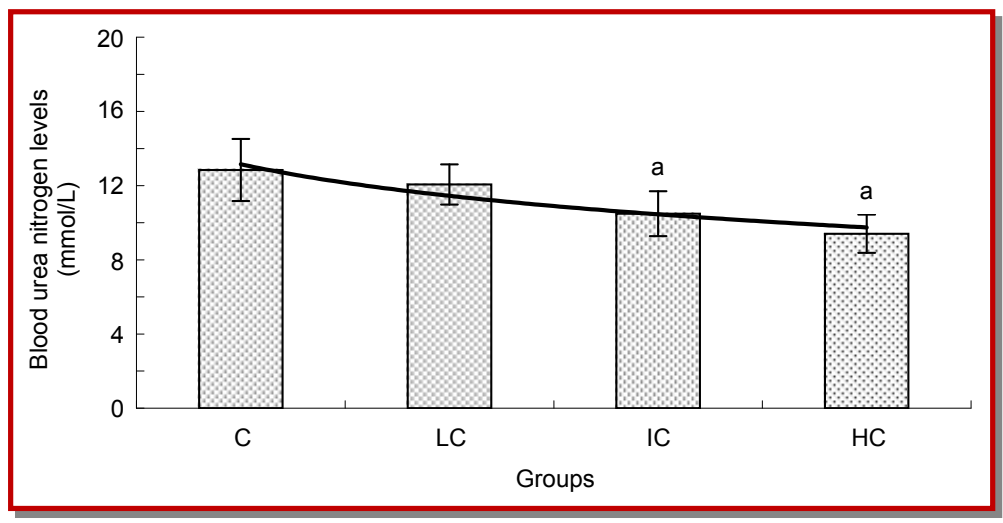

Figure 3: The effects of CSP on the blood urea nitrogen levels of mice. Data are expressed as mean \pm S.D. ap $<0.05$ compared with control group

capacity (Tang et al., 2008). As shown in Table I, the hepatic glycogen and muscle glycogen levels of mice in the LC, IC and HC groups were significantly higher compared with that in the $C$ group $(\mathrm{p}<0.05)$. The results indicated that the anti-fatigue effects of CSP might be related to the improvement in the metabolic control of exercise and the activation of energy metabolism.

\begin{tabular}{|c|c|c|}
\hline \multicolumn{3}{|c|}{ Table I } \\
\hline \multicolumn{3}{|c|}{$\begin{array}{l}\text { The effects of CSP on the hepatic glycogen and } \\
\text { muscle glycogen levels of mice }\end{array}$} \\
\hline Groups & $\begin{array}{c}\text { Hepatic glycogen } \\
(\mathrm{mg} / \mathrm{g})\end{array}$ & $\begin{array}{c}\text { Muscle glycogen } \\
(\mathrm{mg} / \mathrm{g})\end{array}$ \\
\hline Control & $6.5 \pm 1.0$ & $1.2 \pm 0.2$ \\
\hline Low-dose CSP & $11.4 \pm 1.3^{\mathrm{a}}$ & $2.2 \pm 0.3^{a}$ \\
\hline $\begin{array}{l}\text { Intermediate- } \\
\text { dose CSP }\end{array}$ & $14.7 \pm 1.3^{a}$ & $2.6 \pm 0.3^{a}$ \\
\hline High-dose CSP & $16.4 \pm 1.6^{\mathrm{a}}$ & $2.8 \pm 0.4^{\mathrm{a}}$ \\
\hline
\end{tabular}

Data are expressed as mean \pm SD. ${ }^{a} \mathrm{p}<0.05$ compared with control (C) group

The present data suggested that CSP could extend the exhaustive swimming times of mice, as well as increase the hepatic glycogen and muscle glycogen levels, and decrease the blood lactic acid and BUN levels. These results indicated that CSP had anti-fatigue effects. However, further research needs to be carried out to evaluate its anti-fatigue effects at cellular and molecular levels.

\section{Acknowledgements}

This work was supported by a key program of the Bureau of Science and Technology of Harbin, China. We are grateful to Dr. Yiyang Zhang (Jilin University, China) for his revision and comments on the manuscript.

\section{References}

Ataka S, Tanaka M, Nozaki S, Mizuma H, Mizuno K, Tahara T, Sugino T, Shirai T, Kajimoto Y, Kuratsune H, Kajimoto O, Watanabe Y. Effects of applephenon and ascorbic acid on physical fatigue. Nutrition 2007; 23: 419-23.

Chen YQ, Hu B, Xu F, Zhang W, Zhou H, Qu LH. Genetic variation of Cordyceps sinensis, a fruit-body-producing entomopathogenic species from different geographical regions in China. FEMS Microbiol Lett. 2004; 230: 153-58.

Ding JF, Li YY, Xu JJ, Su XR, Gao X, Yue FP. Study on effect of jellyfish collagen hydrolysate on anti-fatigue and antioxidation. Food Hydrocoll. 2011; 25: 1350-53.

Dong $\mathrm{CH}$, Xie XQ, Wang XL, Zhan Y, Yao YJ. Application of Box-Behnken design in optimisation for polysaccharides extraction from cultured mycelium of Cordyceps sinensis. Food Bioprod Process. 2009; 87: 139-44.

Evans WJ, Lambert CP. Physiological basis of fatigue. Am J Phys Med Rehabil. 2007; 86: S29-46.

Huang LZ, Huang BK, Ye Q, Qin LP. Bioactivity-guided fractionation for anti-fatigue property of Acanthopanax senticosus. J Ethnopharmacol. 2011; 133: 213-39.

Ikeuchi M, Koyama T, Takahashi J, Yazawa K. Effects of astaxanthin supplementation on exercise-induced fatigue in mice. Biol Pharm Bull. 2006; 29: 2106-10.

Ji DB, Ye J, Li CL, Wang YH, Zhao J, Cai SQ. Antiaging effect of Cordyceps sinensis extract. Phytother Res. 2009; 23: 116-22.

Jin G, Kataoka Y, Tanaka M, Mizuma H, Nozaki S, Tahara T, Mizuno K, Yamato M, Watanabe Y. Changes in plasma and tissue amino acid levels in an animal model of complex fatigue. Nutrition 2009; 25: 597-607.

Kuo MC, Chang CY, Cheng TL, Wu MJ. Immunomodulatory effect of exo-polysaccharides from submerged cultured Cordyceps sinensis: Enhancement of cytokine synthesis, $\mathrm{CD} 11 \mathrm{~b}$ expression, and phagocytosis. Appl Microbiol Biotechnol. 2007; 75: 769-75. 
Li SP, Zhang GH, Zeng Q, Huang ZG, Wang YT, Dong TT, Tsim KW. Hypoglycemic activity of polysaccharide, with antioxidation, isolated from cultured Cordyceps mycelia. Phytomedicine 2005; 13: 428-33.

Tan W, Yu KQ, Liu YY, Ouyang MZ, Yan MH, Luo R, Zhao $X S$. Anti-fatigue activity of polysaccharides extract from radix Rehmanniae preparata. Int J Biol Macromol. 2012; 50: 5962.

Tang W, Zhang Y, Gao J, Ding X, Gao S. The anti-fatigue effect of 20(R)-ginsenoside Rg3 in mice by intranasally administration. Biol Pharm Bull. 2008; 31: 2024-27.

Tharakan B, Manyam BV. Botanical therapies in chronic fatigue. Phytother Res. 2006; 20: 91-95.

Wang JJ, Shieh MJ, Kuo SL, Lee CL, Pan TM. Effect of red mold rice on antifatigue and exercise-related changes in lipid peroxidation in endurance exercise. Appl Microbiol Biotechnol. 2006; 70: 247-53.

Wang Y, Wang M, Ling Y, Fan W, Wang Y, Yin H. Structural determination and anti-oxidant activity of a polysaccharide from the fruiting bodies of cultured Cordyceps sinensis. Am J Chin Med. 2009; 37: 977-89.

Wu YL, Sun CR, Pan YJ. Studies on isolation and structural features of a polysaccharide from the mycelium of an Chinese edible fungus (Cordyceps sinensis). Carbohydr Polym. 2006; 63: 251-56.

Xu ZX, Wang CF. Effects of radix puerariae (Ge Gen) water extract on exercise-induced fatigue in mice. J Anim Vet Adv. 2012; 11: 1722-26.

Yu F, Lu S, Yu F, Feng S, McGuire PM, Li R, Wang R. Protective effects of polysaccharide from Euphorbia kansui (Euphorbiaceae) on the swimming exercise-induced oxidative stress in mice. Can J Physiol Pharmacol. 2006; 84: 1071-79.

Yu FR, Liu Y, Cui YZ, Chan EQ, Xie MR, McGuire PP, Yu FH. Effects of a flavonoid extract from Cynomorium songaricum on the swimming endurance of rats. Am J Chin Med. 2010; 38: 65-73.

Zhang J, Yu Y, Zhang Z, Ding Y, Dai X, Li Y. Effect of polysaccharide from cultured Cordyceps sinensis on immune function and anti-oxidation activity of mice exposed to 60Co. Int Immunopharmacol. 2011; 11: 2251-57.

Zhang XL, Ren F, Huang W, Ding RT, Zhou QS, Liu XW. Antifatigue activity of extracts of stem bark from Acanthopanax senticosus. Molecules 2010; 16: 28-37.

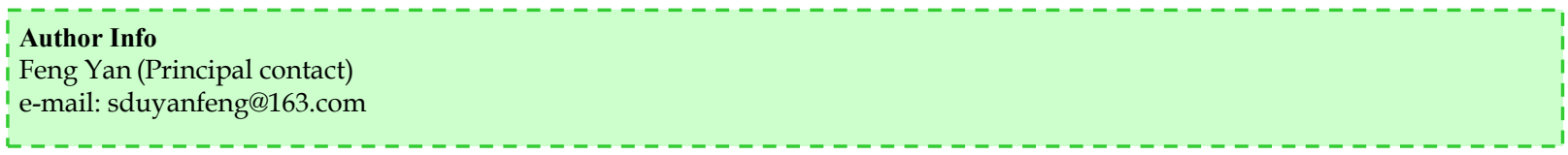

